\title{
Topology Control with Anisotropic and Sector Turning Antennas in Ad-hoc and Sensor Networks
}

\author{
V. Černý
}

\begin{abstract}
During the last several years, technological advances have allowed the development of small, cheap, embedded, independent and rather powerful radio devices that can self-organise into data networks. Such networks are usually called ad-hoc networks or, sometimes, depending on the application field, sensor networks. One of the first standards for ad-hoc networks to impose itself as a fully industrial framework for data gathering and control over such devices is IEEE 802.15.4 and, on top of it, its pair network architecture: ZigBee. In the case of multiple radio devices clamped into a small geographical area, the lack of radio bandwidth becomes a major problem, leading to multiple data losses and unnecessary power drain from the batteries of these small devices. This problem is usually perceived as interference. The deployment of appropriate topology control mechanisms (TC) can solve interference. All of these algorithms calculate $\mathrm{TC}$ on the basis of isotropic antenna radiation patterns in the horizontal plane.
\end{abstract}

Keywords: ad-hoc network simulation, interference, antenna radiation patterns, mobile network simulation, Omnet ++ , topology control, interference, sector antennas, rotating antennas, wireless networks, anisotropic antennas, sensor networks.

\section{Introduction}

Event simulators such as Omnet++ were developed in order to study interference without the deployment of large and sometimes expensive networks. However, these simulators lack the proper radio propagation characteristics that are crucial for understanding the interference phenomenon. The widely-used radio propagation model is isotropic (in the horizontal plane we can imagine it as a circle with the transmitter in the center). This radio pattern is very hard to achieve in the real world. In addition, this model is unusable for simulating the radio patterns of sector antennas.

Classical TC is based on graph theory alone, and does not take into account antenna radiation patterns, propagation, receiver sensitivity or any other radio-related characteristics. Such algorithms, e. g. Relative Neighbourhood Graph [1], Gabriel Graph [1], Yao Graph [1], Minimal Spanning Tree, XTC, ITC [2], etc. are based on the following assumptions: all nodes are situated on a flat surface, no radio propagation model is involved (coverage being determined on geometric properties only), antennas are perfect emitters (isotropic radio pattern), and the receiver is a perfect receiver with no minimal sensitivity threshold. Power regulation is modeled by varying (with infinitely fine increments and with no maximal limit) the radius of the circle that defines the radiation pattern. Such simplified models are easy to test, but they are very far removed from real- ity. To obtain more realistic output it is necessary to involve new transmitter properties in our simulation.

\section{A better antenna simulation model}

For our simulation we do not need the whole 3D antenna propagation pattern because we work only in the horizontal plane, hence flat pattern is sufficient. The modeling is performed by sampling the signal power around the modeled transmitter. The correctness of such a model can by adjusted by changing the size of the sampling step. All measured received power values are recomputed to gain values [3].

In order to obtain an antenna model that is easy to understand and adapt, the radiation pattern for each antenna is defined in Omnet++ as an XML file. Each simulated node can be coupled with one or more modeled antennas; in this way, studies of spatial diversity can be performed easily, with little or no modification to the model itself. Below is a sample code of an antenna radiation pattern:

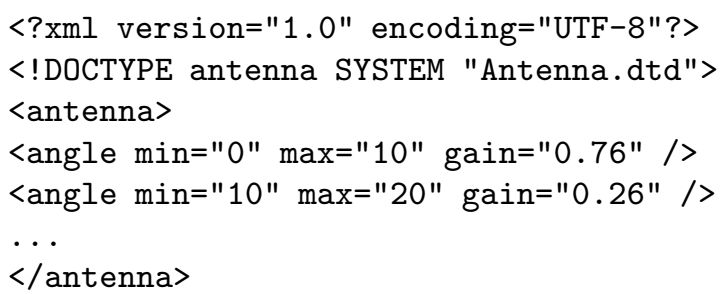


The code was shortened due to the length of the file. This slicing into 10-degree sectors gives good results for reasonable amounts of run time and for the algorithm. The radiation pattern for the above antenna (using the complete set of data) is provided in Figure 1 and Figure 2.

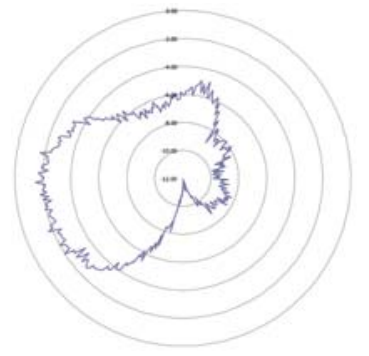

Fig. 1: Gain distribution pattern of a rubber-duck antenna measured in the antenna chamber

\section{TC algorithms with anisotropic antennas}

This section contains the adapted (towards anisotropy) versions of the classical topology control algorithms.

\subsection{ARNG - Anisotropic Relative Neighbourhood Graph}

The subgraph $G^{\prime}=A R N G(G)=\left(V, E^{\prime}\right)$ obtained from graph $G$, where $E^{\prime}$ is:

$$
\begin{aligned}
E(G)^{\prime}= & \{(p, q) \in V \times V \mid u \in V \backslash\{p, q\}, \\
& u \notin \sigma(p) \cap \sigma(q)\}
\end{aligned}
$$

Where $\sigma(p)$ and $\sigma(q)$ are the irregular shaped coverage areas of the communication nodes, defined as the zone where the signal received by an antenna having gain $0 \mathrm{dBi}$ will be at least the minimal power level $P_{\text {min }}$, when $p$ respectively $q$ acts as a transmitter, Figure 4 .

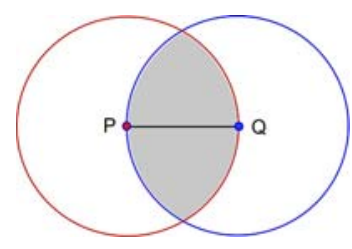

Fig. 3: Step condition in RNG

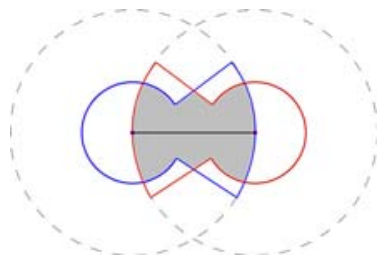

Fig. 4: Step condition in ARNG

\subsection{AGG - Anisotropic Gabriel Graph}

The subgraph $G^{\prime}=A G G(G)=\left(V, E^{\prime}\right)$ obtained from graph $G$, where $E^{\prime}$ is:

$$
\begin{gathered}
(p, q) \in E^{\prime} \text { iff } \Gamma_{p, q} \cap V \backslash\{p, q\} \cap \sigma(p) \cap \sigma(q)=\emptyset \\
\Gamma_{p, q}=D\left(\frac{p+q}{2}, \frac{\delta(p, q)}{2}\right)
\end{gathered}
$$

Put into words, the zone represented by the intersection of the disc centred in the middle of the segment $(p, q)$ and with as its diameter the Euclidian distance between $p$ and $q$, intersected by the irregular coverage areas of $p$ and $q$ must be empty in order for the edge $(p, q)$ to be included in the $E^{\prime}$ set, Figure 6 .

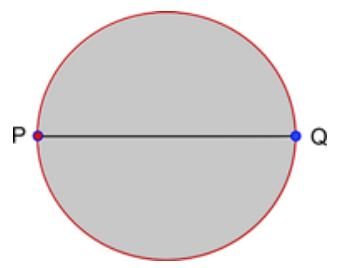

Fig. 5: Step condition in GG

\subsection{AYG - Anisotropic Yao Graph}

The subgraph $G^{\prime}=A Y G(G)=\left(V, E^{\prime}\right)$ obtained from graph $G$, where $E^{\prime}$ is:

$$
\begin{aligned}
& (p, q) \in E^{\prime} i f f \delta(p, q) \leq \\
& \leq\left(\min _{v \in C_{p, q \backslash\{p, q\}}}\{\delta(p, v)\}\right) \wedge(q \in \sigma(p))
\end{aligned}
$$

Put into words, in each sector around node $p$ we choose to connect to the closest node $q$ that lies within the irregular coverage area of node $p$; even though node $r$ is in the same sector closer to $p$ than $q$, node $p$ will not connect to it if $r$ is outside the coverage area of $p$, Figure 8 (the arrow represents the node to connect to, and not an oriented edge).

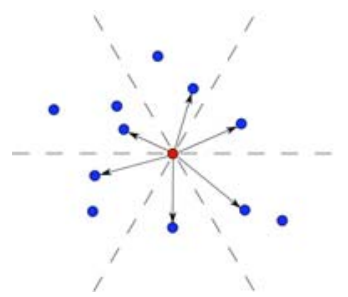

Fig. 7: Step condition in YG

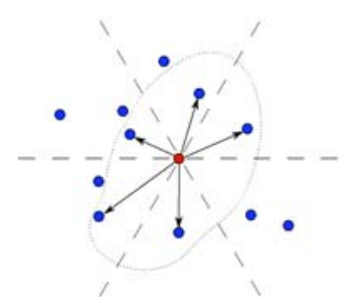

Fig. 8: Step condition in AYG

\subsection{Examples of simulated networks}

Table 1 presents the differences between classical and anisotropic structures for a network containing a uniform random placement of 50 communication nodes. For the anisotropic simulations, the chosen antenna type was rubber-duck; this is motivated by the fact that it has the radiation pattern closest to the ideal dipole. It can be clearly seen that even in this case there are (minor) differences. 
Table 1: Comparison between classical and anisotropic topology control algorithms

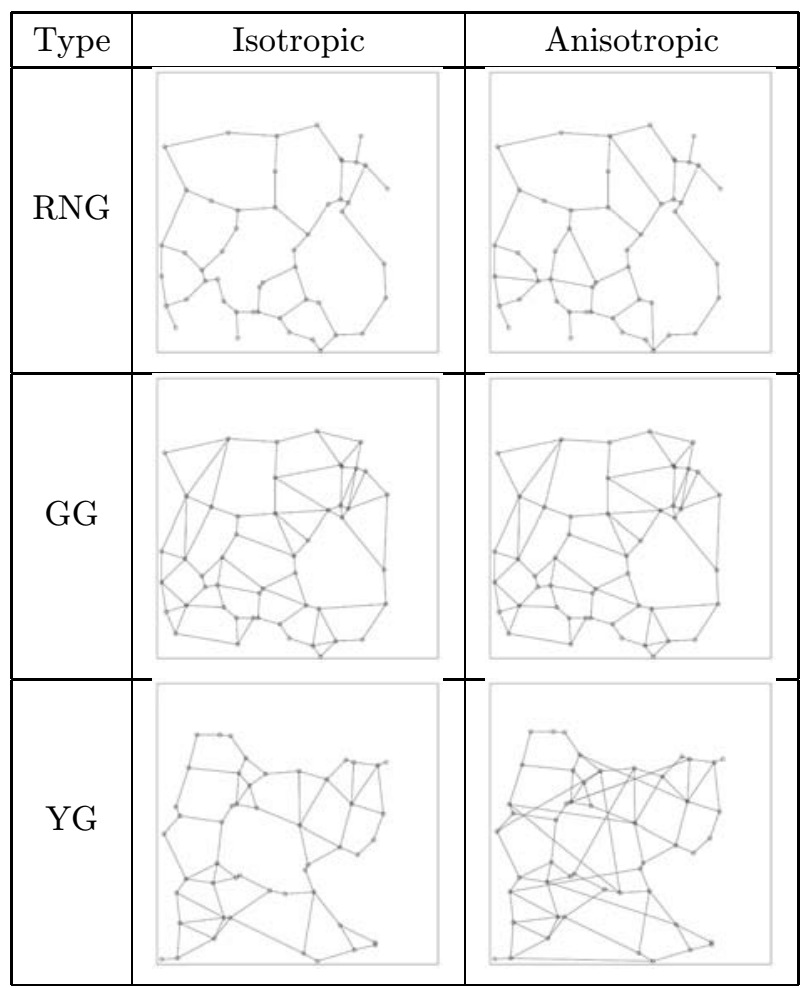

\section{Particular case: high gain sector turning antennas}

A sector turning antenna " $i$ " is defined as a touple $S T A_{i}\left(x, y, G_{1}, G_{2}, \varphi, \theta, P, A, P_{\min }, P_{\max }, P r_{\min }\right.$, $\left.P i_{\text {min }}, \Delta \theta_{\text {min }}, \Delta P_{\text {min }}\right)$, by the following parameters $\langle$ in brackets is the measure unit $\rangle$ :

- coordinates in plane: $x\langle\mathrm{~m}\rangle, y\langle\mathrm{~m}\rangle$;

- gains: $G_{1}\langle\mathrm{dBi}\rangle, G_{2}\langle\mathrm{dBi}\rangle$; with $G_{2}>G_{1}$;

- opening of the main lobe: $\varphi\langle\mathrm{deg}\rangle$;

- rotation of the symmetry axes of the main lobe towards north (azimuth/azimuthal angle): $\theta\langle\operatorname{deg}\rangle$

- power injected in the antenna: $P\langle\mathrm{dBm}\rangle$;

- antenna aperture: $A\left\langle\mathrm{~m}^{2}\right\rangle$;

- minimal and maximal power levels: $P_{\min }\langle\mathrm{dBm}\rangle$ and $P_{\max }\langle\mathrm{dBm}\rangle$, respectively;

- minimal power threshold required for proper data reception: $P r_{\min }\langle\mathrm{dBm}\rangle$;

- minimal power threshold beyond which interference is registered: $P i_{\min }\langle\mathrm{dBm}\rangle$;

- azimuth angle resolution: $\Delta \theta_{\min }\langle\operatorname{deg}\rangle$, with the condition $\Delta \theta_{\text {min }} \leq \varphi$ (in order to cover all the area around the antenna through rotation);

- power resolution: $\Delta P_{\min }\langle\mathrm{dBm}\rangle$;

- $i$ is the index to keep a record of the antennas in the network.

In this model, parameters $x, y, G_{1}, G_{2}, \varphi, A$, $P_{\min }, P_{\max }, P r_{\min }, P i_{\min }, \Delta \theta_{\min }$ and $\Delta P_{\min }$ are fixed (antennas do not move in a plane and do not modify the radiation lobe parameters, the limit power levels or the minimal variation intervals for power or turn).

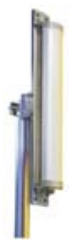

a)

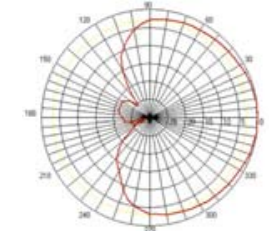

b)

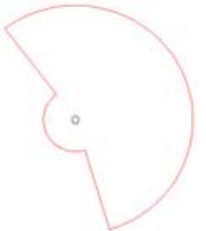

c)
Fig. 9: Example of a $2.4 \mathrm{GHz}$ sector antenna. 1a - real antenna; $1 \mathrm{~b}$ - radiation pattern; $1 \mathrm{c}$ - simulated radiation pattern

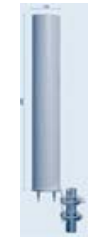

a)

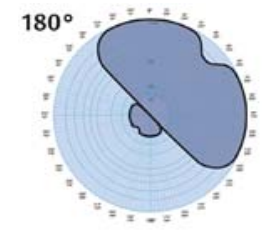

b)

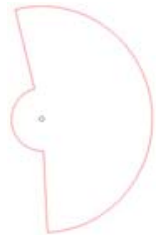

c)
Fig. 10: Example of a Ferimex sector antenna. 1a - real antenna; $1 \mathrm{~b}$ - radiation pattern; $1 \mathrm{c}-$ simulated radiation pattern

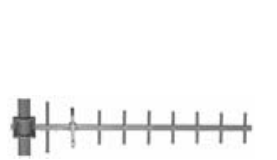

a)

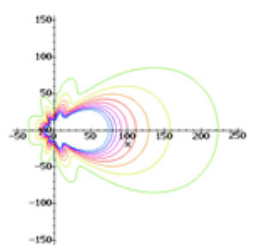

b)

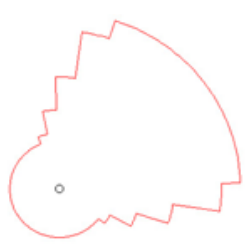

c)
Fig. 11: Example of a Yaggi-Uda lobe antenna. 1a - real antenna; $1 \mathrm{~b}$ - radiation pattern; $1 \mathrm{c}$ - simulated radiation pattern

\section{1 n-Sector turning antenna}

A $n$-sector turning antenna is an extended $S T A$, a touple: $\quad n S T A_{i}\left(x, y, G[], \varphi[], \theta, P, A, P_{\min }, P_{\max }\right.$, $\left.P r_{\text {min }}, P i_{\text {min }}, \Delta \theta_{\text {min }}, \Delta P_{\text {min }}\right)$, where all the parameters excepting $\mathrm{G}[]$ and $\varphi[]$ have the same definition as in the STA definition. $G[]$ represents the gain vector $G[]=G_{1}, G_{2}, \ldots, G_{n-1}, G_{n}, G_{n-1}, \ldots, G_{2}, G_{1}$, where $G_{1}<G_{2}<G_{n}$ and $\varphi[]$ represents the openings vector $\varphi[]=\varphi_{1}, \ldots, \varphi_{n}$, where $\varphi_{j}$ is the angle opening of the zone having gain $G_{j}$. This type of antenna mimics the behaviour of lobe-antennas such as Yaggi-Uda antennas (Figure 11).

\subsection{Network composed of turning sector antennas}

A cluster composed of sector turning antennas is defined by a touple $K\left(n_{k}, X_{k}, Y_{k}, x_{k}, y_{k}, S T A[k]\right)$, where:

- geographical extent of cluster: $X_{k}\langle\mathrm{~m}\rangle, Y_{k}\langle\mathrm{~m}\rangle$;

- geographical coordinates of the centre: $x_{k}\langle\mathrm{~m}\rangle$, $y_{k}\langle\mathrm{~m}\rangle$; 
- number of antennas $n_{k}\langle\rangle$;

- $S T A_{1}, S T A_{2}, \ldots, S T A_{n k}$ component antennas.

All the parameters defining a cluster remain constant (clusters do not change positions or number of components).

A network composed of clusters of sector turning antennas is defined as a touple $N(X, Y, n, k, K[k], Q)$, by the following parameters:

- geographical extent of the network: $X\langle\mathrm{~m}\rangle, Y\langle\mathrm{~m}\rangle$;

- $n\langle\rangle$ number of antennas;

- $k\langle\rangle$ number of clusters;

- $K_{1}, K_{2}, \ldots, K_{k}$ component clusters;

- a property $Q$ that has to be satisfied;

- $n_{k}[1]+n_{k}[2]+\ldots+n_{k}[k]=n$.

A network consisting in a single cluster will have $k=1, X_{k}[1]=X, Y_{k}[1]=Y$ and $n_{k}[1]=n$ - like for example a uniform distribution of nodes over the entire field. Different node placements were tested: random placement - nodes are placed randomly around the geographic network centre (defined by coordinates $[X / 2, Y / 2])$ and cluster placement - the centre of each cluster is chosen randomly around the network centre and the nodes composing one cluster are placed randomly around the centre of the respective cluster. All the parameters defining a network remain constant (the number of clusters in the network, positions of the clusters, etc.). For network $N$, let us define an evaluating metric $E$, which will be detailed in the next paragraph.

Symmetric network: network $N$ is defined as symmetric, thus all the nodes in the network have the same shared parameters $\left(G_{1}, G_{2}, \varphi, A, P_{\min }, P_{\max }\right.$, $P r_{\text {min }}, P i_{\text {min }}, \Delta \theta_{\text {min }}$ and $\Delta P_{\text {min }}$, which is all with the exception of power and rotation of the main lobe towards north). Such a network can model for example a city-wide radio network which consists of identical sector antennas and in which are allocated a very limited number of radio channels. The operator of this network will be interested to connect the entire network (if possible) with low interference rather than low power (because the antennas - in such a network - are powered from the electric grid).

Applying TC to network $\mathrm{N}$ translates to finding an assignment of power and azimuth to north $(P$ and $\theta)$ for each antenna in the network, such that the property $Q$ is achieved (if possible) and the value of evaluation $E$ is improved towards other methods. Classical TC is reduced to finding just a power level assignment $(P)$ for each node in the network such that property $Q$ is fulfilled while $\mathrm{E}$ is evaluated.

\section{Connectivity and interference}

This section defines the connectivity between two nodes and the methods for measuring interference in the network.

\subsection{Connectivity between two nodes}

In the horizontal plane (which in our case corresponds to the magnetic $\mathrm{H}$-plane of all antennas), in the far field, an ideal dipole radiator $l\langle\mathrm{~m}\rangle$ in length, which is fed an alternative current with frequency $f\langle\mathrm{~Hz}\rangle$ and intensity $I\langle\mathrm{~A}\rangle$ will generate a power density (measured by the time-averaged Poynting vector) at distance $r\langle\mathrm{~m}\rangle$ from the radiator $P_{\text {dens }}\left\langle\mathrm{W} / \mathrm{m}^{2}\right\rangle$ given by:

$$
P_{\text {dens }}=\frac{1}{2}\left(\frac{I l}{2 r \lambda}\right)^{2} \eta
$$

where $\eta\langle\Omega\rangle=120 \pi$ is the impedance of the free space and $\lambda\langle\mathrm{m}\rangle=c / f$ is the radio signal wavelength ( $c$ being the speed of light) [3]. In our case $\lambda=0.12244 \mathrm{~m}$ (for $f=2450 \mathrm{MHz}$ ).

Considering the emitter antenna as $S T A_{1}$ and the receiver as $S T A_{2}$, the receiving antenna captures the power density on the whole aperture (A), thus inducing power $P_{\text {ind }}\langle\mathrm{W}\rangle$ equal to the power density multiplied by the aperture of the receiver. However this does not take into account antenna gains; the received power $P_{\text {recv }}\langle\mathrm{dBm}\rangle$, with the gain contribution, becomes [3]:

$$
\begin{aligned}
P_{\text {recv }}\langle\mathrm{dBm}\rangle= & P_{\text {ind }}\langle\mathrm{dBm}\rangle+G_{12}\langle\mathrm{dBi}\rangle+ \\
& G_{21}\langle\mathrm{dBi}\rangle-F S P L\langle\mathrm{~dB}\rangle
\end{aligned}
$$

where $P_{\text {ind }}$ had to be converted from W to dBm, $G_{12}$ is the gain of the emitter facing towards the receiver, $G_{21}$ is the gain of the receiver towards the emitter, and FSPL is the free space path loss of the connection [3]. In order to have communication between $S T A_{1}$ and $S T A_{2}$ it is required that $P_{\text {recv }} \geq P r_{\text {min }}$.

Thus, the minimal power density level that must be created by antenna $S T A_{1}$ to communicate to $S T A_{2}$ can be computed as:

$$
P_{\text {dens }}\left\langle\mathrm{W} \cdot \mathrm{m}^{-2}\right\rangle=\frac{10^{\frac{P r_{\min }-G_{12}-G_{21}}{10}}}{A}
$$

by converting $\mathrm{dBm}$ to $\mathrm{W}$ and where $A\left\langle\mathrm{~m}^{2}\right\rangle=3 \lambda^{2} / 8 \pi$ is the aperture of the receiver (dipole-based sector antenna).

The power that must be injected into the emitter $S T A_{1}$ can be calculated by integrating the power density $P_{\text {dens }}$ over the whole surface that is the $3 \mathrm{D}$ radiation pattern of the antenna. Thus $P\langle\mathrm{~W}\rangle=$ $P_{\text {dens }}\left\langle\mathrm{W} / \mathrm{m}^{2}\right\rangle \mathrm{S}\left\langle\mathrm{m}^{2}\right\rangle$. In the case of dipole-based sector antennas or lobe antennas (like Yaggi-Uda), the radiation surface is $S=8 \pi r^{2} / 3$ and in the case of an ideal antenna (the radiation pattern of which is a perfect sphere) is $S=4 \pi r^{2}$, with $r$ in this case being the distance between the two antennas.

In this paper the minimal receive level was chosen at $P r_{\min }=-46.511 \mathrm{dBm}$, based on the XBee Series 
2 modules specification (however it can be chosen at any other value, depending on the used network devices) and the required antenna gains were chosen on the basis of various sector and Yaggi-Uda antennas.

\section{Interference in the network}

Based on the radio parameters, six interference estimations are defined (for node, edge, maximal and average cases); interference is the metric $E$ that will be evaluated for each network.

For module $S T A_{1}$, let us define the radio coverage interference of the node as the number of other nodes that receive from $S T A_{1}$ a radio signal stronger than $P i_{\min }$ :

$$
\begin{aligned}
& \operatorname{Rcov}\left(S T A_{1}\right)= \\
& \left|\begin{array}{c}
\left\{S T A_{x} \in N \backslash S T A_{1}\right. \\
\left.P_{\text {recv }}\left(S T A_{1} \rightarrow S T A_{x}\right) \geq P i_{\min }\right\}
\end{array}\right|
\end{aligned}
$$

where $P_{\text {recv }}\left(S T A_{1^{-}}>S T A_{x}\right)$ designates the power induced by node $S T A_{1}$ at the $S T A_{x}$ node.

For bi-directional communication between $S T A_{1}$ and $S T A_{2}$, let us define the radio coverage interference of the whole link $S T A_{1}-S T A_{2}$ as the number of other nodes that receive a radio signal stronger than $P i_{\text {min }}$ from either $S T A_{1}$ or $S T A_{2}$ :

$$
\begin{aligned}
& R \operatorname{cov}\left(S T A_{1}-S T A_{2}\right)= \\
& \left|\begin{array}{c}
\left\{S T A_{x} \in N\left\{S T A_{1} ; S T A_{2}\right\} ;\right. \\
\left.P_{\text {recv }}\left(S T A_{1} \rightarrow S T A_{x}\right) \geq P i_{\min }\right\} \\
\cup\left\{S T A_{y} \in N\left\{S T A_{1} ; S T A_{2}\right\}\right. \\
\left.P_{\text {recv }}\left(S T A_{2} \rightarrow S T A_{y}\right) \geq P i_{\min }\right\}
\end{array}\right|
\end{aligned}
$$

For the whole network, let us define the maximal and average radio interference values, based on either nodes (vertices) or links (edges in a graph representing the network):

$$
\begin{aligned}
& R I V \operatorname{Cov}(N)=\max _{S T A_{x} \in N}\left(\operatorname{RCov}\left(S T A_{x}\right)\right. \\
& R I E \operatorname{Cov}(N)= \\
& \max _{S T A_{x}-S T A_{y}}\left(R \operatorname{cov}\left(S T A_{x}-S T A_{y}\right)\right)
\end{aligned}
$$

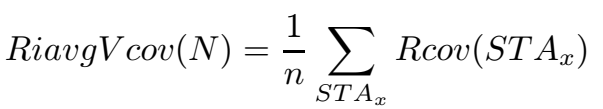

$$
\begin{aligned}
& R I \operatorname{Avg} \operatorname{Ecov}(N)= \\
& \frac{1}{n} \sum_{S T A_{x}-S T A_{y}} \operatorname{Rcov}\left(S T A_{x}-S T A_{y}\right)
\end{aligned}
$$

where $n$ is the number of all nodes in the network. In all the simulated networks $P i_{\min }$ was chosen at $-55 \mathrm{dBm}$, based on the XBee specification (it can be chosen at other values, depending on the used network devices). The $E$ estimation in this paper was chosen as RIAvgECov. From (5) it can be seen that the radio signal decreases with the square of the distance from the emitter - corresponding to the freespace propagation model.

\section{$7 \quad$ TC with sector turning antennas}

Two algorithms are presented in this paper: MaxDistanceMinimise (MDistM) and axDegreeMinimise (MDegM). The two algorithms do not require global information about the network (each node relies on local node discovery); each of the two algorithms consists in two stages/steps: discovery and configuration; because the discovery phase is common it will be presented only once. If global information about the network is available in the form of geographical coordinates of the antennas, the first step (discovery) can be skipped.

\subsection{Node Discovery}

This phase assumes that each antenna is randomly oriented at initial azimuthal angle $\theta_{i}$ and it requires one complete turn in the azimuthal plane in order to perform a full discovery. It is also required that a neighbour antenna should not be identified more than once. For this purpose, the antennas exchange Hello messages that contain: a unique id (antenna index $i$ ), current azimuth $\theta$ (read from an internal compass, for example) - based on this the receiver can determine whether the sender is using the strongest radiation lobe. The algorithm requires $360 / \Delta \theta_{\min }$ rotation steps to complete.

Node Discovery (all nodes execute in parallel):

- set power level to maximum $P=P_{\max }$;

- while $\theta<\theta_{i}+360$ execute:

- exchange Hello packets;

- identify neighbours reachable with azimuth $\theta$;

- $\theta=\theta+\Delta \theta_{\min }$

- build a neighbour table NT containing: neighbour id., possible azimuths $\theta$ that each neighbour can be reached with, and the power level that each neighbour was listened to;

- exclude from NT the cases when a neighbour can be seen only if both antennas are oriented with the strongest lobe towards each other. These neighbours are very distant and they will be disconnected;

\subsection{MaxDistanceMinimise (MDistM)}

This algorithm is based on the fact that links between distant nodes can be achieved by orienting a stronger lobe of radiation in the desired direction, without increasing the power injected into the emitter. The 
algorithm requires maximum $\left(P_{\max }-P_{\min }\right) / \Delta P_{\min }$ steps of power adjustment to complete.

MaxDistanceMinimise (all nodes execute in parallel):

- determine the most distant neighbour (MDN) from the NT (MDN is the node that has the lowest received signal over all registered entries in NT);

- calculate the azimuth $\theta_{x}$ to reach the $M D N$;

- set power level to maximum $P=P_{\max }$;

- while $\theta<\theta_{x}$ execute: $\theta=\theta+\Delta \theta_{\min }$;

- while $P<P_{\min }$ execute:

- exchange Hello messages;

test connectivity with neighbours in $N T$;

- if no nodes missing $P=P-\Delta P_{\min }$;

- else $P=P+\Delta P_{\min }$ and end while cycle;

- while $P>P_{\min }$ execute:

- exchange NT tables with neighbours;

- test connectivity with neighbours through intermediate nodes (acting as relays);

- if no nodes missing $P=P-\Delta P_{\min }$;

- else $P=P+\Delta P_{\min }$ and end while cycle.

- Result: $\left(\theta_{x}, P\right)$ for each node.

\subsection{MaxDegreeMinimise (MDegM)}

This algorithm is based on the fact that in clustered networks it is desired that the main radiation lobe will be used to create intercluster links, while the weak lobe will be used to create intracluster links. The algorithm requires maximum $\left(P_{\max }-P_{\min }\right) / \Delta P_{\min }$ steps of power adjustment to complete.

The degree of a transmitter node in one direction $\theta$ is adapted from graph theory, and is defined as the number of other nodes that can receive a signal above $P r_{\text {min }}$ from the transmitter if the main radiation lobe of the transmitter has azimuthal angle $\theta$.

MaxDegreeMinimise (all nodes execute in parallel):

- from NT calculate the azimuth $\theta_{x}$ that corresponds to the direction for which the transmitter has the highest degree;

- set power level to maximum $P=P_{\max }$;

- while $\theta<\theta_{x}$ execute: $\theta=\theta+\Delta \theta_{\min }$;

- while $P>P_{\min }$ execute:

- exchange Hello messages;

- test connectivity with neighbours in NT;

- if no nodes missing $P=P-\Delta P_{\min }$;

- else $P=P+\Delta P_{\min }$ and end while cycle;

- while $P>P_{\min }$ execute:

- exchange NT tables with neighbours;

- test connectivity with neighbours through intermediate nodes (acting as relays);

- if no nodes missing $P=P-\Delta P_{\min }$;

- else $P=P+\Delta P_{\min }$ and end while cycle. Result: $\left(\theta_{x}, P\right)$ for each node.

\section{Power level adjustment}

The algorithms MDistM and MDegM presented in the previous sections make use of linear power decrease, which is the standard approach for having power adjustments implemented in hardware. However faster results can be achieved if the power levels are adjusted exponentially:

PowAdj:

- set power level to maximum $P=P_{\max }$;

- base $b=2$; exponent $e=1$;

- while $P>P_{\min }$ execute:

- $\Delta P=\frac{P_{\max }}{b^{e}}$

- exchange NT tables with neighbours;

- test connectivity with neighbours through intermediate nodes (acting as relays);

- if no nodes missing $P=P-\Delta P$;

- else $P=P+\Delta P_{\min }$ and end while cycle;

- $e=e+1$.

\section{Simulation results}

For the simulations, we chose a squared flat playground of $180 \times 180 \mathrm{~m}$ on which 20 or 50 nodes (equipped with STA or nSTA, uniform networks all nodes have one type of antenna only) were placed in different configurations: uniform random and clustered. A cluster placement is defined by choosing the number of clusters (2 to 10) and their geometric sizes ( 5 to $25 \mathrm{~m}$ ): the centres of the clusters are placed randomly around the playground centre and the nodes of each cluster are placed within the borders of the cluster randomly around the centre of the cluster.

The following experiments were performed for both 20 and 50 nodes, uniform or clustered, STA or nSTA:

- sector opening $(\varphi)$ variation: the gains $G_{1}$ and $G_{2}$ are maintained constant, while $\varphi$ is increased from $10^{\circ}$ to $350^{\circ}$;

- gain $\left(G_{2}\right)$ variation: the gain $G_{1}$ and the sector opening $\varphi$ are maintained constant, while the gain $G_{2}$ is increased from $2 \mathrm{dBi}$ to $20 \mathrm{dBi}$;

- sector openings for nSTA with 5 to 10 gain zones in the gain vector, ranging from $1.5 \mathrm{dBi}$ to $20 \mathrm{dBi}$.

In order to compare the interference results, we used the same simulator as in [1], the same configurations for node distributions and wave propagation. In the following graphs we compare the results obtained by applying anisotropic TC (ARNG, AGG, AYG — without antenna turning) with results obtained by antenna turning. Finally, the total power need per network is estimated for all the methods in order to keep the network connected. Each presented result is an average over 100 simulations of the same type; a total of 52000 networks were simulated for this paper. 


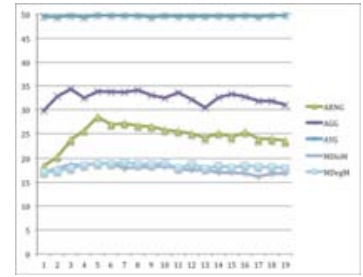

a) Interference $(0 y)$, uniform distribution

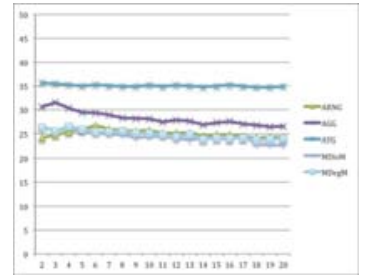

b) Total power $(0 y-d B m)$, uniform distribution
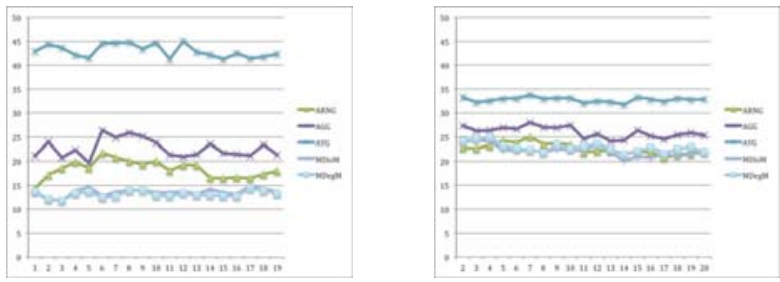

d) Total power $(0 y-d B m)$, cluster distribution

Fig. 12: 50 nodes STA gain increment for sector $\varphi=10^{\circ}, G_{1}=1.5 \mathrm{dBi}, G_{2}=2 \ldots 20 \mathrm{dBi}(0 x)$

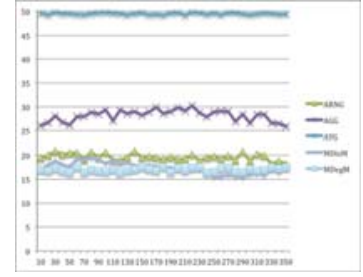

a) Interference $(0 y)$, uniform distribution

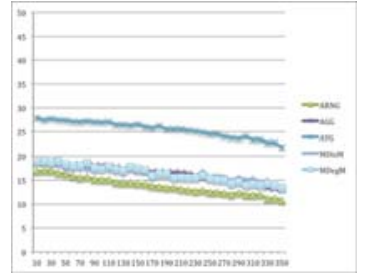

b) Total power $(0 y-d B m)$, uniform distribution

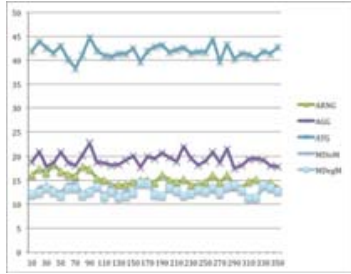

c) Interference $(0 y)$, cluster distribution

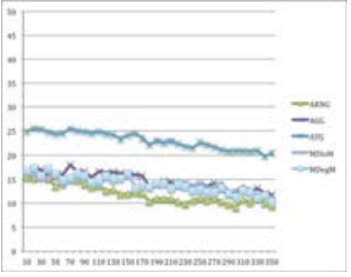

d) Total power $(0 y-d B m)$, cluster distribution

Fig. 13: 50 nodes STA sector increment $\varphi=10^{\circ} \ldots 350^{\circ}(0 x)$ for gains $G_{1}=3.5 \mathrm{dBi}, G_{2}=7 \mathrm{dBi}$

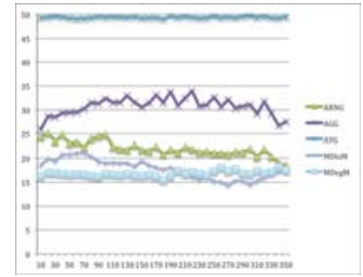

a) Interference $(0 y)$, uniform distribution

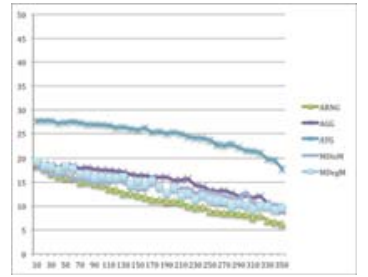

b) Total power $(0 y-d B m)$, uniform distribution

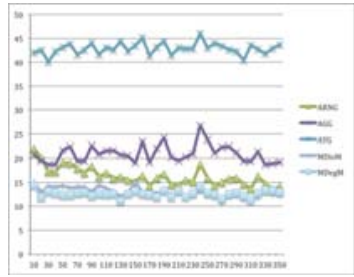

c) Interference $(0 y)$ cluster distribution

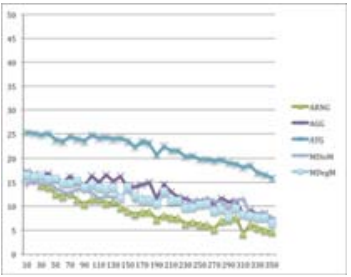

d) Total power $(0 y-d B m)$, cluster distribution

Fig. 14: 50 nodes STA sector increment $\varphi=10^{\circ} \ldots 350^{\circ}(0 x)$ for gains $G_{1}=3.5 \mathrm{dBi}, G_{2}=12 \mathrm{dBi}$

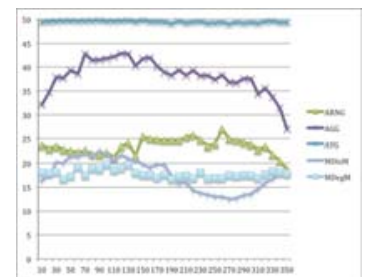

a) Interference $(0 y)$, uniform distribution

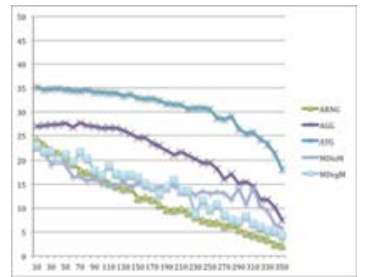

b) Total power $(0 y-d B m)$, uniform distribution

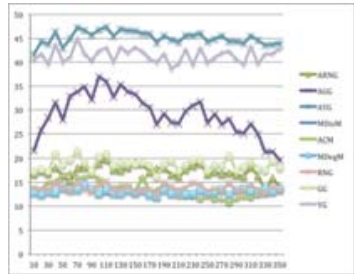

c) Interference $(0 y)$, cluster distribution

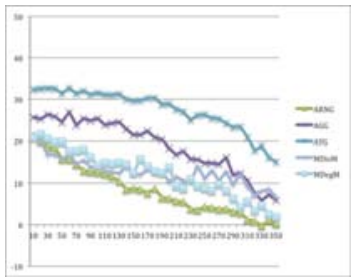

d) Total power $(0 y-d B m)$, cluster distribution

Fig. 15: 50 nodes STA sector increment $\varphi=10^{\circ} \ldots 350^{\circ}(0 x)$ for gains $G_{1}=3.5 \mathrm{dBi}, G_{2}=20 \mathrm{dBi}$ 


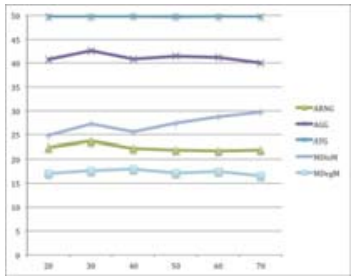

a) Interference $(0 y)$, uniform distribution

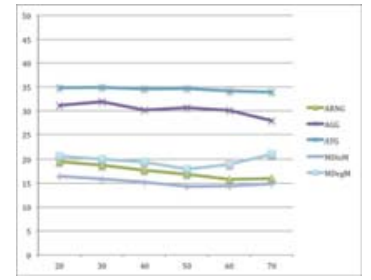

b) Total power $(0 y-d B m)$, uniform distribution

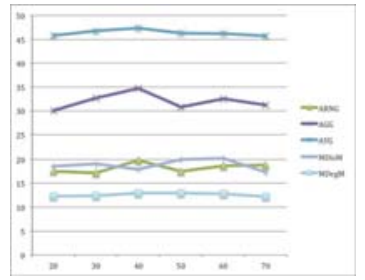

c) Interference $(0 y)$, cluster distribution

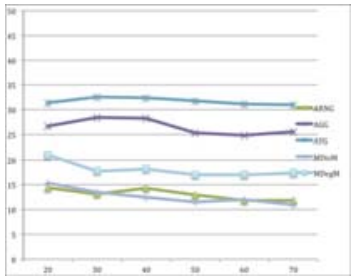

d) Total power $(0 y-d B m)$, cluster distribution

Fig. 16: 50 nodes nSTA sector increment $n=5, \varphi_{1}=\varphi_{2}=\varphi_{3}=\varphi_{4}=10^{\circ}, \varphi_{5}=20^{\circ} \ldots 70^{\circ}(0 x), G_{1}=2 \mathrm{dBi}$, $G_{2}=4 \mathrm{dBi}, G_{3}=8 \mathrm{dBi}, G_{4}=16 \mathrm{dBi}, G_{5}=20 \mathrm{dBi}$

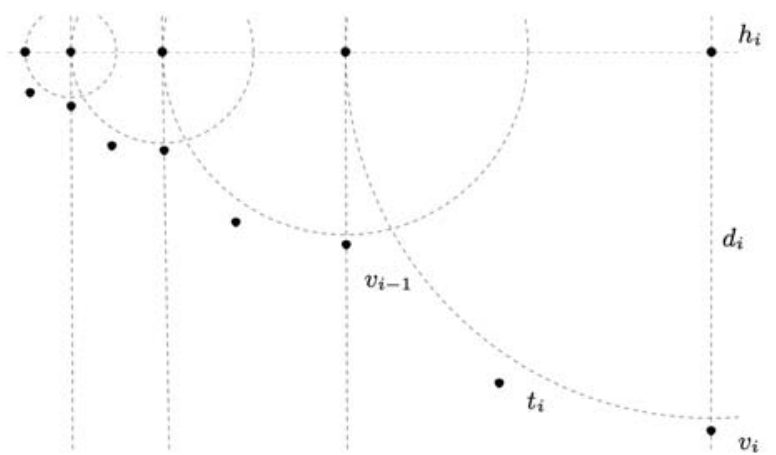

a) Exponential node chain and solution to have lower interference: introduction of auxiliary nodes

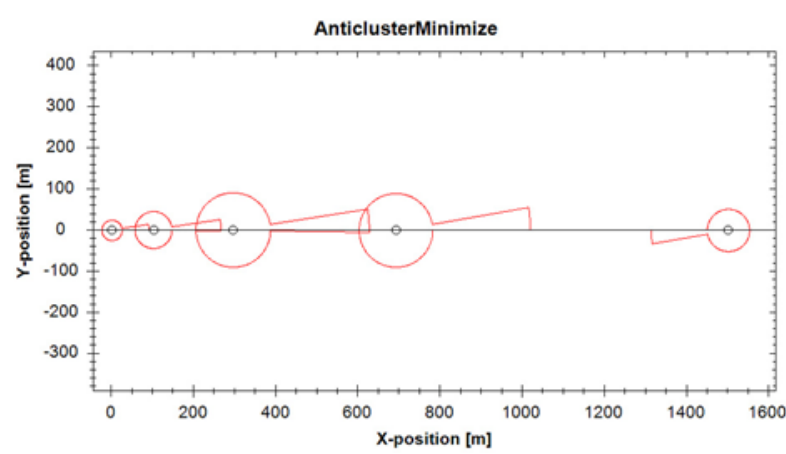

b) Solution with sector antennas: no auxiliary nodes required, zero additional interference. Note that in this image the radiation pattern is correlated to power not coverage - thus a link can exist even when a node is not located inside the radiation pattern

Fig. 17: Exponential node chain

\section{Conclusions}

As is shown in Figures 12-16, MDistM and MDegM have better behaviour (lower interference and power need) for cluster distributions than for uniform distribution: this is explained by the fact that a high-gain lobe tends to create intercluster links and a residual lobe tends to create intracluster links. Another consideration is the decrease in power need when the main lobe widens (Figures 13-15b and d).

Another example architecture is the exponential node chain - all nodes placed on the same axes with distances increasing exponentially (Figure 17a. on axes $0 x$ ) [4]; in this case, the use of isotropic antennas yields huge interference values (this being in fact a counterexample for low node degree TC, which does not ensure low interference). However, the node topology can be elegantly solved by using STA (Figure 17b.), where each antenna has gain $G_{2}=2 G_{1}$, which proves that antenna behavior opens new horizons for TC.
Nodes which take advantage of using the new TC with STA are capable to create networks that need less energy consumption. As it can be seen, the networks created in such a way model very well WiFi infrastructure networks for metropolitan areas (for example, networks composed by turning high-gain antennas placed on tops of building roofs). What it is presented in this paper is a network which is capable to automatically connect and reconnect in case of a failure (due to the removal of one communication node for example).

The principle described in this paper is interesting because it can be improved, for example by combining TC with smart antennas [3]. Different TC methods which take into account slightly different parameters as an input will generate slightly different results, suitable for different real-world scenarious. How to precisely choose what is best for each scenario is a subject for future improvement in this area. 


\section{References}

[1] Wagner, D., Wattenhofer, R.: Algorithms for Sensor and Ad Hoc Networks - Advanced Lectures, ISSN 0302-9743, Springer, 2007, pp. 85-90.

[2] Janecek, J., Moucha, A.: Comparison of local interference-aware topology control algorithms, Proceedings of the 19th IASTED International Conference on Parallel and Distributed Computing and Systems, 2007. ISBN 978-0-88986-704-8.

[3] Balanis, C.: Antenna Theory - Analysis and Design. 3rd ed., Wiley, 2005, pp. 154-162. ISBN 0-471-66782-X.

[4] Burkhart, M., von Rickenbach, P., Wattenhofer, R., Zollinger, A.: Does Topology Con- trol Reduce Interference?, MobiHoc '04: Proceedings of the 5th ACM international symposium on Mobile ad hoc networking and computing, 2004, pp. 9-19.
About the author
Viktor ČERNÝ was born in Prague in 1982 and was awarded his master degree in 2009. Currently he is a $\mathrm{PhD}$ student in computer science at FEL CVUT.
Viktor Černý
E-mail: cernyvi2@fel.cvut.cz
Dept. of Computer Science and Engineering Faculty of Electrical Engineering
Czech Technical University
Technická 2, 16627 Praha, Czech Republic 